\title{
The Influence of Different Type Irradiations on the Surface States Parameters of $\mathrm{Si}^{-\mathrm{SiO}_{2}}$ Structures
}

\author{
Aram A. Sahakyan 1, Hrant N. Yeritsyan ${ }^{1 *}$, Vachagan V. Harutunyan"1, Hamlet S. Karayan², \\ Vahan A. Sahakyan ${ }^{3}$ \\ ${ }^{1}$ A. I. Alikhanyan National Science Laboratory, Yerevan Physics Institute, Yerevan, Armenia \\ ${ }^{2}$ Yerevan State University, Yerevan, Armenia \\ ${ }^{3}$ National Institute of Metrology, Yerevan, Armenia \\ Email: grant@yerphi.am
}

Received 8 July 2015; accepted 21 September 2015; published 24 September 2015

Copyright (C) 2015 by authors and Scientific Research Publishing Inc.

This work is licensed under the Creative Commons Attribution International License (CC BY). http://creativecommons.org/licenses/by/4.0/

(c) (i) Open Access

\section{Abstract}

The results of experimental research of some effects in metal-insulator-semiconductor (MIS) structures induced by different types of radiation $(50 \mathrm{MeV}$ electrons, $12 \mathrm{keV}$ gamma-quanta, 10 and $40 \mathrm{keV}$ arsenic ions) are presented. It is found that there is a significant difference between the characters of radiation surface states (SS) formed by ionization and impact actions of the MIS structure irradiation at the insulator-semiconductor (I-S) interface. It is shown that the SS generation rate is increased in electric fields and depends on the MIS structure field electrode material.

\section{Keywords}

Metal-Insulator-Semiconductor (MIS) Structures, Radiation Effects, Surface States (SS) Density, Insulator-Semiconductor (I-S) Interface, Annealing

\section{Introduction}

Recent progress in nanotechnology has led to the development of a wide range of quantum and low-dimensional nanoelectronic, nanooptic and optoelectronic devices. Semiconductor nanolasers operating at room temperature, quantum computers based on two-dimensional electron gases in MIS structures, etc. are already known [1]-[3]. Their energy spectrum and optical properties, as well as other physical properties determined by the structure of

${ }^{*}$ Corresponding author.

How to cite this paper: Sahakyan, A.A., Yeritsyan, H.N., Harutunyan, V.V., Karayan, H.S. and Sahakyan, V.A. (2015) The Influence of Different Type Irradiations on the Surface States Parameters of $\mathrm{Si}_{-} \mathrm{SiO}_{2}$ Structures. Journal of Modern Physics, 6, 1657-1662. http://dx.doi.org/10.4236/imp.2015.611167 
the system itself and by a number of external (control) fields and impacts are quite well studied. Due to their unique properties and advanced features, optical quantum devices are candidates to become major components of the space measurement, communications and automation systems.

However, the phenomena of influence of ionizing radiation and other penetrating exposures are still not well understood. Any quantum, such as optical (for example, nanolasers), or information (for example, MIS structure) system contains several metal-semiconductor, metal-insulator and insulator-semiconductor interfaces, at which external damaging impacts are rather different. Some of them, for example, space charges formed in dielectric layers, can be estimated by the Schrödinger equation, however an increase in the SS density at the insulatorsemiconductor (I-S) interface was studied insufficiently. At the same time the surface states strongly affect the kinetics of physical processes in a system, reduce the carrier lifetime, increase noise, etc. In some optoelectronic quantum computers the information is coherently transferred over a distance on the following principle: "the first qubit state-intricate laser field state in resonator - the second qubit state". Surface states accelerate the loss of coherence of processes, thereby violating the unitary character of computations, as well as the control accuracy.

At present comprehensive information on radiation effects in MIS structures and their impact on physical characteristics of these structures is available [4]-[8]. The main part of the radiation defects introduced in semiconductor structures is electrically active and forms energy levels that play an important role in the formation of electro-physical parameters of insulator-semiconductor (I-S) structures.

The surface radiation defects are formed both under the influence of heavy ions, high-speed electrons, gamma-quanta irradiation and low-energy electrons (with energies below the threshold of elastic displacement of atoms in crystals), even at X-ray or ultraviolet treatment with the energy below $5 \mathrm{eV}$ [6] [8] [9].

This paper presents some results of comparative study of parameters of radiation surface states generated on the $\mathrm{SiO}_{2}$-Si interface under different types of irradiation with significantly different mechanisms of interaction between the incident particles and I-S components.

\section{Experimental Results and Discussion}

In order to investigate the influence of mechanisms of interaction between the incident particles and I-S components on the radiation SS parameters, the following types of radiation were selected—pure ionization: synchrotron radiation (SR) with the quanta characteristic energy of $12 \mathrm{keV}$ (Figure 1), mainly ionization: $50 \mathrm{MeV}$ fast electrons, and impact-ionization: 10 and $40 \mathrm{keV}$ energy arsenic ions with a projected range in $\mathrm{SiO}_{2}$ about $10 \mathrm{~nm}$ and $24 \mathrm{~nm}$, respectively. The irradiation by arsenic ions was carried out from the oxide site of $\mathrm{Si}_{-} \mathrm{SiO}_{2} \mathrm{sample}_{\text {. }}$

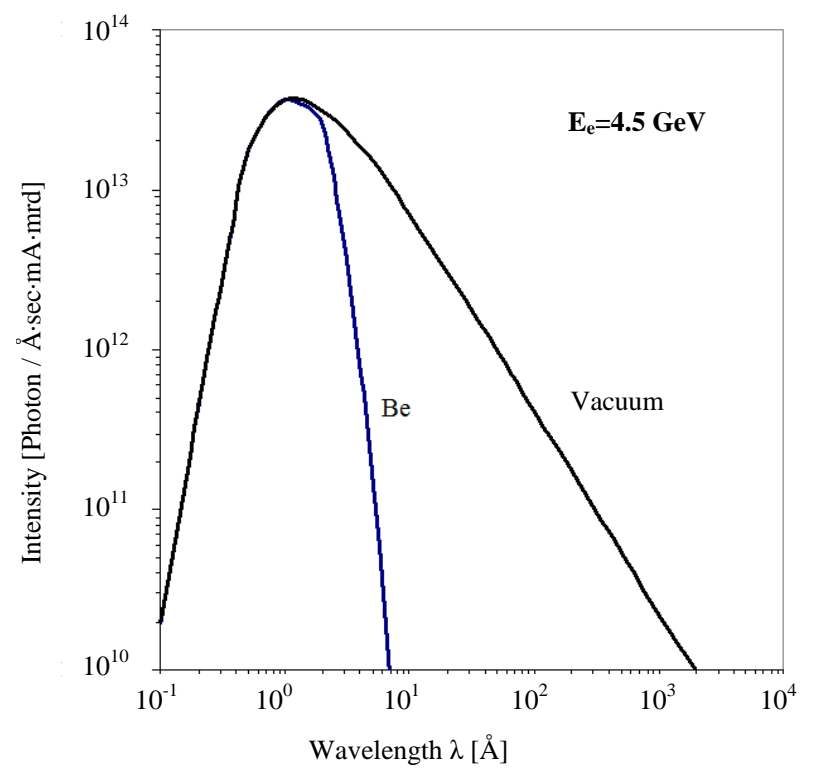

Figure 1. Calculated spectral intensity distribution of synchrotron radiation with the electron energy of $E_{e}=4.5 \mathrm{GeV}$ : through a beryllium window in the air $(\mathrm{Be})$ and in a vacuum chamber. 
All kinds of radiation exposure were carried out at room temperature. The parameters of the surface centres located from the n-Si midgap to the Fermi level were measured by conductance technique [10], whereas the parameters of those located from the Fermi level to the conductivity band edge-by capacitance technique. M$\mathrm{SiO}_{2}$-Si structures with thermal oxidation having oxide thickness of $85-100 \mathrm{~nm}$, grown on a crystallographic plane [10] on the n-type silicon with an electron density of $5 \times 10^{14} \mathrm{~cm}^{-3}$ in the bulk crystal were studied. Since under operating mode the MIS devices are under the action of electric fields, it is also important to study the influence of electric fields on the formation of radiation-induced defects at the I-S interface.

Different opinions have been expressed in several papers devoted to the influence of electric fields on the generation of radiation SS in MIS structures. For example, it is stated in [7] that the SS accumulation rate can be greatly affected by the external electric field when exposing to UV sources with positive polarity on the field electrode, whereas in the case of negative polarity a slight increase in the SS density is observed. Other authors have found that both positive and negative voltages affect the accumulation rate of surface centers when exposing MOS structures to UV radiation.

Figure 2 shows SS spectra after electron irradiation in the presence of electric fields of various strength and polarity. It is seen that under the influence of electric fields, the introduction rate of radiation SS significantly increases, and the field polarity does not exert a pronounced influence on SS generation. It also shows a weak dependence of the radiation SS introduction rate on the electric field magnitude.

In our opinion, the obtained data can be attributed to the fact that during irradiation, holes and/or electrons generated by the radiation contribute to the SS accumulation. They diffuse to the I-S interface under the action of the external electric field from oxide (in the case of positive electrode voltage) or from semiconductor (in the case of negative electrode voltage). It is important to note that no special features are observed in the spectrum of energy dependence of the density of the SS created in the presence of electric fields and without them, and these curves are qualitatively identical.

Figure 3 shows the energy spectra of radiation surface states $N_{s s}(E)$, generated by different types of highenergy particles with different mechanisms of interaction with MIS structure components. It is seen that SS spectra generated by $50 \mathrm{MeV}$ electrons and synchrotron radiation with the characteristic length of about $0.1 \mathrm{~nm}$ (maximum quantum energy of about $12 \mathrm{keV}$ ) have identical shapes, and therefore it can be assumed that in both cases the surface centers have similar nature. However, bombardment of $\mathrm{SiO}_{2}$ structure by short-range 10 and 40 $\mathrm{keV}$ arsenic ions creates surface centers with spectrum significantly different from those of SS formed under fast electron and synchrotron radiation influence.

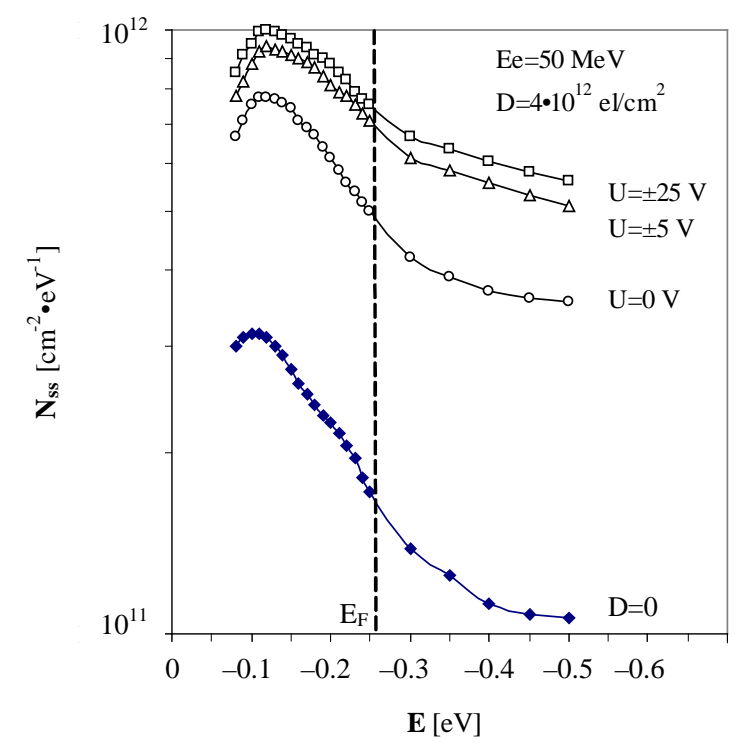

Figure 2. Energy dependence of the radiation SS density $N_{\text {ss }}(E)$ after $50 \mathrm{MeV}$ electron irradiation of $\mathrm{Al}-\mathrm{Si}-\mathrm{SiO}_{2}$ structure (at a dose of $\mathrm{D}=4 \times 10^{12} \mathrm{el} / \mathrm{cm}^{2}$ ): $\mathrm{D}=0$ before irradiation; $\mathrm{U}=0$, irradiated without electric field; $\mathrm{U}= \pm 5 \mathrm{~V}$; $\mathrm{U}= \pm 25 \mathrm{~V}$-when applying electric field to the electrode at the same dose. $\mathrm{E}_{\mathrm{F}}-$ Fermi level in Si volume. 


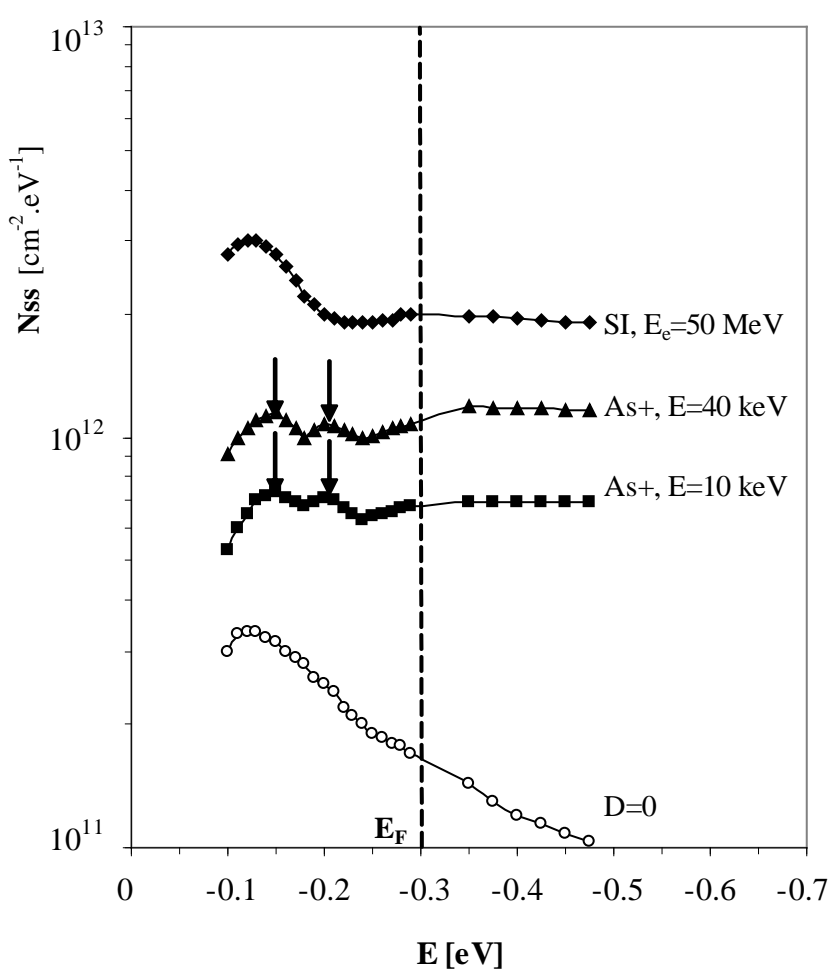

Figure 3. Energy dependence of the radiation SS density $N_{s s}(E)$ on $\mathrm{SiO}_{2}$-Si structure irradiated by: $10 \mathrm{keV}$ and $40 \mathrm{keV}$ arsenic ions with a projected range in $\mathrm{SiO}_{2}$ of about $10 \mathrm{~nm}$ and 24 $\mathrm{nm}$, respectively, with a dose of $1 \mu \mathrm{Ku} / \mathrm{cm}^{2}$; SI-synchrotron radiation; $50 \mathrm{MeV}$ electrons at a dose of $10^{13} \mathrm{el} / \mathrm{cm}^{2}$. Arrows on curves indicate the density peaks of SS caused by radiation defects with the energetic levels in the Si forbidden gap of $\mathrm{E}_{\mathrm{c}}-0.15 \mathrm{eV}$ and $\mathrm{E}_{\mathrm{c}}-0.21 \mathrm{eV}$.

It is seen that in the case of predominantly ionization radiation, SS with a continuous energy spectrum are generated with density increasing at the beginning, when approaching from the Fermi level to the conduction band edge, $E_{c}-0.12 \mathrm{eV}$, and then decreasing. As for the impact mechanism of action, besides SS with a continuous spectrum, two peak densities are observed over this background at $E_{c}-0.15 \mathrm{eV}$ and $E_{c}-0.21 \mathrm{eV}$. These data allow assuming that ion bombardment results in the formation of surface centers that are different in nature.

This assumption is also confirmed by the study of isochronous annealing of radiation $\mathrm{SS}$ in $\mathrm{SiO}_{2}$-Si structures (see Figure 4). The curves of isochronal annealing of the surface traps generated by the ionization mechanism of radiation interaction have a single stage with a median temperature of $\mathrm{t} \approx 140^{\circ} \mathrm{C}$ and annealing activation energy of $\varepsilon_{\mathrm{A}}=0.85 \pm 0.1 \mathrm{eV}$. Whereas for the impact interaction mechanism isochronal annealing curve has two stages with median temperatures of $\mathrm{t}_{1} \approx 180^{\circ} \mathrm{C}$ and $\mathrm{t}_{2} \approx 240^{\circ} \mathrm{C}$ and annealing activation energies of $\varepsilon_{\mathrm{A} 1}=1.7 \pm$ $0.2 \mathrm{eV}$ and $\varepsilon_{\mathrm{A} 2}=2.8 \pm 0.3 \mathrm{eV}$, respectively.

The effect of the field electrode material on the accumulation rate of radiation SS in MIS structures was also studied. For this purpose $\mathrm{Al}, \mathrm{Au}, \mathrm{Ag}, \mathrm{Cu}, \mathrm{Zn}$, Ni metals deposition by vacuum evaporation on the different points of the same sample was used. Figure 5 presents the SS energetic spectra for the case of $50 \mathrm{MeV}$ electron irradiation at a dose of $3 \times 10^{13} \mathrm{el} / \mathrm{cm}^{2}$.

Energy dependence of the radiation SS density $N_{\text {ss }}(E)$ of $\mathrm{M}-\mathrm{Si}_{-}-\mathrm{SiO}_{2}$ structure with a field electrode from different materials irradiated by $50 \mathrm{MeV}$ electrons $\left(\mathrm{D}=3 \times 10^{13} \mathrm{el} / \mathrm{cm}^{2}\right)$. All metal electrodes were deposited on $\mathrm{SiO}_{2}$ by vacuum evaporation; $\mathrm{Al}^{*}$ was deposited after irradiation.

It is seen from the Figure 5 that the accumulation rate of radiation SS in the case of electron irradiation with aluminum electrode is significantly higher than with other metals. The same figure shows the SS spectral curve of $N_{\text {ss }}(E)$ obtained from the identical irradiated $\mathrm{Si}_{-} \mathrm{SiO}_{2}$ sample, when the $\mathrm{Al}^{*}$ field electrode was deposited after electron irradiation. To explain the high accumulation rate of radiation SS, some additional experimental investigations of the samples with different thickness of metal Al electrode and two dielectric electrodes (silicon 


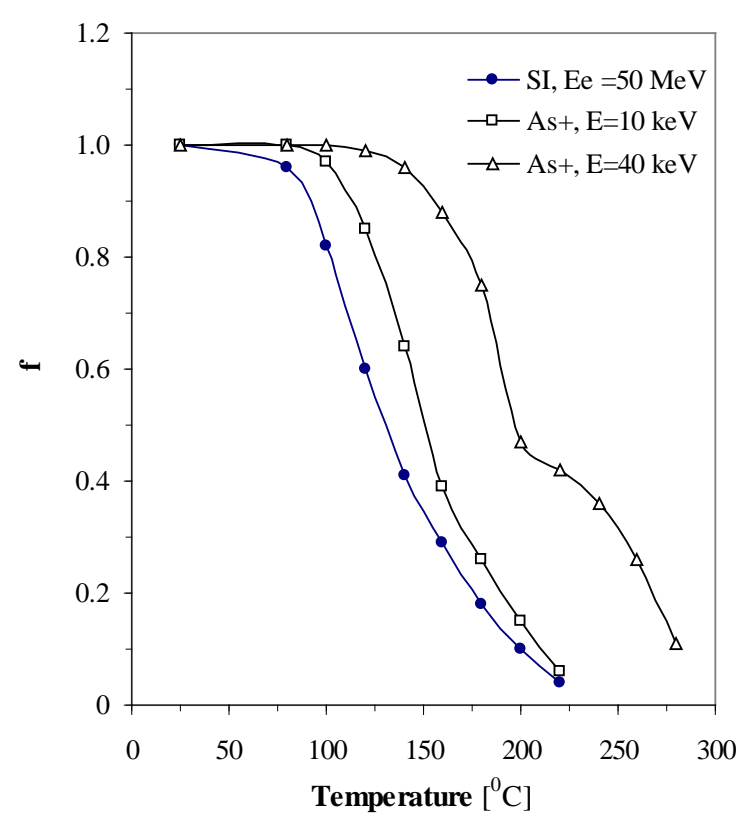

Figure 4. The temperature dependence of the fraction (f) of non-annealed SS at the isochronal annealing of $\mathrm{SiO}_{2}-\mathrm{Si}$ structure, irradiated by synchrotron radiation and electrons with $50 \mathrm{MeV}$ energy $\left(\mathrm{D}=3 \times 10^{13} \mathrm{el} / \mathrm{cm}^{2}\right)$ and arsenic ions with energy of $10 \mathrm{keV}$ and $40 \mathrm{keV}\left(\mathrm{D}=1 \mu \mathrm{Ku} / \mathrm{cm}^{2}\right) . \quad f=\left(N_{\mathrm{ss}}(T)-N_{\mathrm{ss}}^{0}(T)\right) /\left(N_{\mathrm{ss}}-N_{\mathrm{ss}}^{0}(T)\right): N_{\mathrm{ss}}, \quad N_{\mathrm{ss}}(T)$; SS density before and after annealing, respectively, $N_{s s}^{0}(T)$-the density of the SS control non-irradiated sample. At $50 \mathrm{MeV}$ energy electron and SI irradiations the corresponding curves coincide.

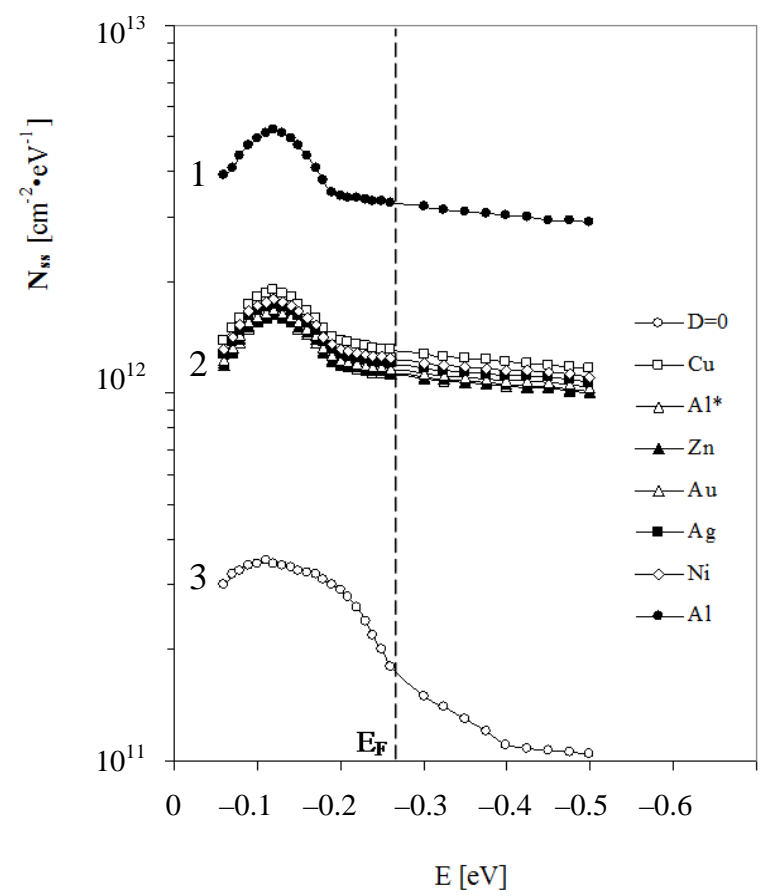

Figure 5. Energy dependence of the radiation SS density $N_{\text {ss }}(E)$ of $\mathrm{M}$-Si-SiO $\mathrm{S}_{2}$ structure with a field electrode from different materials irradiated by $50 \mathrm{MeV}$ electrons $\left(\mathrm{D}=3 \times 10^{13} \mathrm{el} / \mathrm{cm}^{2}\right)$. All metal electrodes were deposited on $\mathrm{SiO}_{2}$ by vacuum evaporation; $\mathrm{Al}^{*}$ was deposited after irradiation: curve 1 represents the energy dependence of the radiation SS density $N_{\text {ss }}(E)$ of $\mathrm{M}-\mathrm{Si}-\mathrm{SiO}_{2}$ structure with $\mathrm{Al}$ electrode, curve 3 corresponds to non-irradiated sample, while curves 2 represent this dependence for other metallic electrodes which are almost the same. 
oxide- $\mathrm{SiO}_{2}$ and silicon nitride- $\mathrm{Si}_{3} \mathrm{~N}_{4}$ ) were carried out. These experimental investigations have shown that the anomalous increase in the generation rate of radiation $\mathrm{SS}$ in the case of aluminum electrode in $\mathrm{Al}-\mathrm{SiO}_{2}$-Si structures is observed only if there is an adhesive bond between aluminum and silicon oxide.

\section{Conclusion}

Comparative study of the parameters of radiation surface centers generated at the $\mathrm{Si}_{-} \mathrm{SiO}_{2}$ interface after irradiation of MOS structure with different types of radiation has shown that, depending on the interaction mechanism between the bombarding particles and MOS structure atoms, radiation surface states of different nature are formed at the insulator-semiconductor interface. The energy spectra, annealing characteristic temperatures and the activation energies of the radiation interface centers depend significantly on the interaction mechanism. Electric fields, regardless of their sign, exert a significant influence on the formation of SS in the I-S system irradiated by fast electrons: they substantially increase the accumulation rate of radiation $\mathrm{SS}$ in $\mathrm{Si}-\mathrm{SiO}_{2}$ structures. It is interesting and important to note that the accumulation rate of radiation SS created in the $\mathrm{M}^{-\mathrm{SiO}_{2}}$-Si structures irradiated with fast electrons in the case of aluminum electrode is higher by a factor of 3 - 4 than that in the case of $\mathrm{Au}, \mathrm{Ag}, \mathrm{Cu}, \mathrm{Ni}, \mathrm{Zn}$.

\section{References}

[1] Zhukov, A.E. (2007) Lasers Based on Semiconductor Nanostructures. St. Petersburg LLC “Tekhnomed”, Moscow, 304. (In Russian)

[2] Dragunov, V.P., Neizvestny, I.G. and Gridchin, V.A. (2006) Fundamentals of Nanoelectronics. Fizmatgiz, Moscow, 496. (In Russian)

[3] Karayan, H.S. (2011) Defining of the Marginal State of a Quantum System at its Conditionally Cyclic Evolution. Proceedings of the International Conference Electron, Positron, Neutron and X-Ray Scattering under the External Influences, Yerevan-Meghri, 215-219. (In Russian)

[4] Mitchell, J.P. and Wilson, D.K. (1967) The Bell System Technical Journal, 46, 1-81.

[5] Sah, C.T. (1976) IEEE Transactions on Nuclear Science, 5-23, 1563-1568.

[6] Eritsyan, G.N., Mordkovich, V.N., Oganessyan, A.S. and Sahakyan, A.A. (1987) Crystal Lattice Defects and Amorphous Materials, 14, 256-260.

[7] Winokur, P.S., McGarrity, L.M. and Boesch, H.E. (1976) IEEE Transactions on Nuclear Science, 5-23, $1580-1585$.

[8] McCaughan, V. (1973) Journal of Applied Physics, 44, 2008-2013.

[9] Parchinsky, P.B., Vlasov, S.I. and Nasirov, A.A. (2004) Semiconductors, 38, 1345-1348.

[10] Nicollian, E.N. and Goetzberger, A. (1967) The Bell System Technical Journal, 46, 1055-1134. 